\title{
Sertraline or placebo in chronic breathlessness? Lessons from placebo research
}

\author{
To the Editor:
}

We were fascinated by the recent paper from CuRRow et al. [1], which described the largest randomised controlled trial of an antidepressant in the treatment of chronic refractory breathlessness. This study was important as there are few pharmacological treatments available for chronic breathlessness. In this group there is an unmet clinical need for treatments that target symptoms. The study's theoretical basis was well supported by preliminary data. However, no difference was observed between sertraline and placebo for the primary outcome measure, the improvement in breathlessness intensity.

We are particularly interested in the observation that breathlessness intensity actually improved across both arms of the study. As described in the results: "At the end of the study, 26 (36.1\%) out of 72 participants on sertraline and $31(41.3 \%)$ out of 75 on placebo felt appreciable improvement... A minority felt sufficient benefit for long-term use (sertraline $18.6 \%$, placebo $26.3 \%$ )".

These observations highlight two clinically relevant points: first, the problem of demonstrating the superiority of a drug over placebo in randomised placebo-controlled trials (RCT), which is the gold standard for proof of efficacy, and second, that there is now increasing realisation about how placebo might be harnessed for clinical benefit.

Placebo responses are well-documented in many conditions including chronic pain, depression and asthma [2]. In randomised controlled trials, placebo response is a well-recognised factor that masks the true pharmacodynamic effects of a drug. This is a particular issue in early small scale phase II studies (proof of concept stage) where some evidence of efficacy is sought before the drug can be advanced to the next stages. Due to the placebo effect, a drug with true pharmacodynamic effects might be dropped early in the development process [3].

Placebo response has a neural basis thought to be related to shaping the way the brain forms expectations [4]. Emerging evidence highlights the role of expectation in the way the brain generates the feelings of breathlessness $[5,6]$. It is possible that drugs acting in the central nervous system can interact with these networks failing to mount the placebo related neural response in the drug arm [7]. This then challenges the validity of the whole premise of randomised controlled trials which assumes that expectation-driven aspects are equal in the drug arm and the placebo arm. Although expectation-driven behavioural components might seem equal, the neural basis that drives the symptomatic improvement in both arms might not be the same in the placebo and the drug arm.

The second point it highlights is the importance of the potential benefits of placebo treatment that is often ignored by medical professionals [8]. The term "placebo" is often used disparagingly to suggest a treatment does not work. Prescribing of placebo is restricted, due to ethical concerns about deception.

A recent upsurge in interest in harnessing placebo response for clinical benefit in the treatment of chronic pain has direct relevance for chronic breathlessness. Some of the ethical concerns have been addressed by the use of "open label" placebo, in which patients are fully aware that they are receiving an inert medicine [8]. The trials explain open label placebo in positive terms, such as "this pill has no active constituents but has been shown to work for some people”. A recent systematic review on open label placebo in several clinical

@ERSpublications

Harnessing the placebo response may benefit patients with chronic breathlessness http://ow.ly/1BTK30naRZc

Cite this article as: Pattinson K, Wanigasekera V. Sertraline or placebo in chronic breathlessness? Lessons from placebo research. Eur Respir J 2019; 53: 1802225 [https://doi.org/10.1183/13993003.02225-2018]. 
conditions indicate a significant beneficial effect when compared to no treatment, though larger clinical trials are needed to confirm this concept [9].

Based on the findings of Currow et al. [1], combined with what is already known about placebo in chronic pain, we feel that this is a sufficient evidence base to support further research on the mechanisms of placebo in chronic breathlessness, the duration of the placebo response and also on the open label placebo concept.

Kyle Pattinson and Vishvarani Wanigasekera

Nuffield Dept of Clinical Neurosciences, University of Oxford, Oxford, UK.

Correspondence: Kyle Pattinson, University of Oxford, Nuffield Dept of Anaesthetics and Oxford Centre for Functional Magnetic Resonance Imaging of the Brain, John Radcliffe Hospital, Oxford, OX3 9BT, UK.

E-mail: kyle.pattinson@nda.ox.ac.uk

Received: Nov 222018 | Accepted: Nov 252018

Conflict of interest: K. Pattinson reports personal fees for consultancy from Nektar Pharmaceuticals, outside the submitted work; and is supported by the National Institute for Health Research, Oxford Biomedical Research Centre based at Oxford University Hospitals NHS Trust and University of Oxford. V. Wanigasekera has nothing to disclose.

\section{References}

1 Currow DC, Ekström M, Louw S, et al. Sertraline in symptomatic chronic breathlessness: a double blind, randomised trial. Eur Respir J 2019; 53: 1801270.

2 Kaptchuk TJ, Miller FG. Placebo effects in medicine. N Engl J Med 2015; 373: 8-9.

3 Hewitt DJ, Hargreaves RJ, Curtis SP, et al. Challenges in analgesic drug development. Clin Pharmacol Ther 2009; 86: $447-450$

4 Ongaro G, Kaptchuk TJ. Symptom perception, placebo effects, and the Bayesian brain. Pain 2019; 160: 1-4.

5 Faull OK, Hayen A, Pattinson KTS. Breathlessness and the body: neuroimaging clues for the inferential leap. Cortex 2017; 95: 211-221.

6 Herigstad M, Faull OK, Hayen A, et al. Treating breathlessness via the brain: changes in brain activity over a course of pulmonary rehabilitation. Eur Respir J 2017; 50: 1701029.

7 Wanigasekera V, Wartolowska K, Huggins JP, et al. Disambiguating pharmacological mechanisms from placebo in neuropathic pain using functional neuroimaging. Br J Anaesth 2018; 120: 299-307.

8 Kaptchuk TJ, Miller FG. Open label placebo: can honestly prescribed placebos evoke meaningful therapeutic benefits? BMJ 2018; 363: k3889.

9 Charlesworth JEG, Petkovic G, Kelley JM, et al. Effects of placebos without deception compared with no treatment: a systematic review and meta-analysis. J Evid Based Med 2017; 10: 97-107.

Copyright @ERS 2019

From the authors:

K. Pattinson and V. Wanigasekera raise important questions about understanding better the placebo response. This effectiveness study randomised people to either double-blind sertraline or placebo [1]. The study reported a response rate in the placebo arm (change in breathlessness now) almost mirroring that for sertraline. However, this observed change in the control group needs to be distinguished from any placebo response. There are at least two factors which are difficult to quantify but are likely to account for the vast majority of improved breathlessness that was seen in the control and intervention arms: the Hawthorne effect [2]; and regression to the mean [3]. Any placebo response is likely to be a very small component of the response rate in the placebo arm.

The Hawthorne effect reflects the changes in participant behaviour (and potentially outcomes) when they know that their actions or responses are being observed. For example, the acknowledgement of each participant's chronic breathlessness and its impact on their lives, a more comprehensive clinical assessment

@ERSpublications

A small proportion of the response in the placebo arm is likely to be attributable to a true "placebo response" http://ow.ly/XLwI30naUWP

Cite this article as: Currow DC, Agar M, Ekström M. Sertraline or placebo in chronic breathlessness? Lessons from placebo research. Eur Respir J 2019; 53: 1802316 [https://doi.org/10.1183/13993003.023162018]. 
as a result of being in a clinical trial, and the increased contact with health professionals during the trial are likely to influence positively people's perceptions and improve their sense of wellbeing [2]. Participants in each arm of a randomised controlled trial (RCT) should undergo exactly the same procedures for the intervention (treatment or control), meaning the Hawthorne effect is likely to affect arms equally.

Regression to the mean reflects that any measure that is at the more extreme level of a scale is more likely to have subsequent measures closer to the mean [4]. Regression to the mean happens widely in biological systems and accounts for much of the response seen in any population with symptoms sufficiently intense to enter a symptom control RCT. Improvements in the control group with intense symptoms should therefore be expected. The RCT is evaluating whether any improvement between arms is greater than the rate of regression to the mean.

Many symptom control studies have had high rates of improvement in the placebo arm documented [5], even where there has been extensive pharmacological pre-treatment of the symptom [6]. Creating a control arm with no additional clinical contact (to deal with the Hawthorne effect) does not account for regression to the mean. This makes it almost impossible to quantify any real placebo response rate.

The authors agree that a placebo response warrants further studies to elucidate the underpinning mechanisms and the potential therapeutic use, but any clinical trial work in this area is likely to be confounded by trial participation and the intensity of symptoms. How such a response can be harnessed therapeutically in severe chronic symptoms is unclear from the available literature. Patients' roles in the co-design of such studies will be crucial to ensure it meets the needs and expectations of patients as any findings are applied in clinical care.

David C. Currow ${ }^{1,2}$, Meera Agar ${ }^{1,3}$ and Magnus Ekström ${ }^{1,4}$

${ }^{1}$ IMPACCT, University of Technology Sydney, Ultimo, Australia. ${ }^{2}$ Wolfson Palliative Care Research Centre, University of Hull, Hull, UK. ${ }^{3}$ Ingham Institute for Medical Research, Liverpool, Australia. ${ }^{4}$ Lund University, Faculty of Medicine, Dept of Clinical Sciences Lund, Respiratory Medicine and Allergology, Lund, Sweden.

Correspondence: David C. Currow, University of Technology, Sydney - IMPACCT, Ultimo, Sydney, New South Wales 2007, Australia. E-mail: david.currow@uts.edu.au

Received: Dec 062018 | Accepted: Dec 092018

Conflict of interest: D.C. Currow is an unpaid member of an advisory board for Helsinn Pharmaceuticals, is a consultant to Specialised Therapeutics and Mayne Pharma and received intellectual property payments from Mayne Pharma. M. Agar has nothing to disclose. M. Ekström has nothing to disclose.

\section{References}

1 Currow DC, Ekström M, Louw S, et al. Sertraline in symptomatic chronic breathlessness: a double blind, randomised trial. Eur Respir J 2019; 53: 1801270.

2 McCarney R, Warner J, Iliffe S, et al. The Hawthorne Effect: a randomised, controlled trial. BMC Med Res Methodol 2007; 7: 30.

3 Bland JM, Altman DG. Statistics notes: some examples of regression towards the mean. Br Med J 1994; 309: 780.

4 Davis CE. The effect of regression to the mean in epidemiologic and clinical studies. Am J Epidem 1976; 104: 493-498.

5 Abernethy AP, McDonald CF, Frith PA, et al. Effect of palliative oxygen versus room air in relief of breathlessness in patients with refractory dyspnoea: a double-blind randomized controlled trial (NCT00327873). Lancet 2010; 376: 784-793.

6 Hardy J, Quinn S, Fazekas B, et al. Randomised, double-blind, placebo-controlled study to assess the efficacy and toxicity of subcutaneous ketamine in the management of cancer pain. J Clin Oncol 2012; 30: 3611-3617. 